\title{
Allele frequencies for 70 autosomal SNP loci with U.S. Caucasian, African-American, and Hispanic samples
}

\author{
Peter M. Vallone*, Amy E. Decker, John M. Butler \\ Biotechnology Division, National Institute of Standards and Technology, \\ Gaithersburg, MD 20899-8311, USA
}

Received 1 June 2004; received in revised form 28 July 2004; accepted 30 July 2004 Available online 23 September 2004

\begin{abstract}
189 samples from 3 different U.S. sample groups Caucasian (74), African American (71) and Hispanic (44) were typed for 70 autosomal genetic markers. These 70 markers are bi-allelic $(\mathrm{C} / \mathrm{T})$ short nucleotide polymorphisms (SNPs). For each sample, the 70 SNP markers were typed in 11 unique 6-plexes and a single 4-plex PCR. A total of 10 of the 210 tests (70 loci $\times 3$ populations) for Hardy-Weinberg equilibrium indicated a statistically significant result. In order to evaluate the minimum number of SNP loci needed to distinguish all 189 samples from one another, we ranked the loci according to their levels of observed heterozygosity and p-values obtained upon testing for Hardy-Weinberg equilibrium. The top 12 loci according to these ranking criteria were tabulated along with the number of unique genotypes observed when combining subsequent SNP markers. The 12 selected SNPs possessed an observed heterozygosity of $>0.45$ in all three populations examined and thus would be expected to exhibit more differences between samples. All of the 189 samples in this study were individualized with a subset of 12 SNP loci. However, it is likely that the addition of more than 12 SNP loci will be required to resolve larger sets of unrelated individuals from one another. By way of comparison, in these same 189 individuals all but one pair is resolved from one another with three of the traditional short tandem repeat (STR) loci possessing the highest heterozygosity values (D2S1338, D18S51, and FGA) run with the Identifiler kit. The final pair of unrelated samples could be resolved with the combination of 4 STR loci: D2S1338, D18S51, FGA, and VWA.

Published by Elsevier Ireland Ltd.
\end{abstract}

Keywords: Single nucleotide polymorphism; DNA; SNP; Autosomal markers; Primer extension

\section{Population samples}

Anonymous liquid blood samples with self-identified ethnicities were purchased from Interstate Blood Bank, Inc. (Memphis, TN) and Millennium Biotech, Inc. (Ft. Lauderdale, FL). All samples were previously examined with 15 autosomal short tandem repeats and the amelogenin

\footnotetext{
* Corresponding author. Tel.: +1 301975 4872; fax: +1 3019758505 .

E-mail address: peter.vallone@nist.gov (P.M. Vallone).
}

sex-typing marker using the AmpFlSTR Identifiler kit (Applied Biosystems, Foster City, CA) to verify that each sample was unique [1].

N: Seventy-four U.S. Caucasians, 71 African-Americans and 44 U.S. Hispanics were typed for 70 autosomal bi-allelic single nucleotide polymorphism (SNP) markers.

\section{DNA extraction}

Blood samples were extracted using a modified salting out procedure [2]. 


\section{Quantification}

Extracted DNA was quantified using UV spectrophotometry followed by a PicoGreen assay [3] to adjust concentrations to approximately $1 \mathrm{ng} / \mu \mathrm{l}$.

\section{SNP markers}

The 70 autosomal SNP markers are listed in Table 1 (see also http://www.cstl.nist.gov/biotech/strbase/SNP.htm). The PCR primer sequences were obtained from Orchid Cellmark (personal communication, Jeanine Baisch, Orchid Cellmark Dallas). The exact chromosomal locations were ascertained using BLAT (http://genome.ucsc.edu/cgi-bin/hgBlat) and dbSNP (http://www.ncbi.nlm.nih.gov/SNP/) and are based on the July 2003 assembly of the human genome. All of the SNPs are $\mathrm{C} / \mathrm{T}$ transitions.

\section{PCR amplification}

For each sample, the 70 SNP markers were typed in 11 unique 6-plexes and a single 4-plex PCR. The final concentrations of the six (or 4) PCR primer pairs were present at $0.5 \mu \mathrm{M}$ for all multiplex PCRs. Amplifications were performed in reaction volumes of $10 \mu \mathrm{l}$ using a master mix containing 1X GeneAmp ${ }^{\mathbb{R}}$ PCR Gold buffer (Applied Biosystems, Foster City, CA), $4.5 \mathrm{mmol} / \mathrm{l} \mathrm{MgCl} 2,250 \mu \mathrm{mol} / \mathrm{l}$ deoxynucleotide triphosphates (dNTPs; Promega Corporation, Madison, WI), $0.16 \mathrm{mg} / \mathrm{ml}$ bovine serum albumin (BSA) fraction V (Sigma, St. Louis, MO), and 0.5 unit of AmpliTaq Gold ${ }^{\mathbb{R}}$ DNA polymerase (Applied Biosystems). The thermal cycling program was carried out on a GeneAmp 9700 (Applied Biosystems) using the following conditions in 9600-emulation mode (i.e., ramp speeds of $1{ }^{\circ} \mathrm{C} / \mathrm{s}$ ) [4]:

\section{$95{ }^{\circ} \mathrm{C}$ for $10 \mathrm{~min}$}

Three cycles of $\left\{95{ }^{\circ} \mathrm{C}\right.$ for $30 \mathrm{~s}, 50{ }^{\circ} \mathrm{C}$ for $55 \mathrm{~s}, 72{ }^{\circ} \mathrm{C}$ for $\left.30 \mathrm{~s}\right\}$

18 cycles of $\left\{95^{\circ} \mathrm{C}\right.$ for $30 \mathrm{~s}, 50{ }^{\circ} \mathrm{C}$ for $30 \mathrm{~s}+0.2^{\circ} \mathrm{C}$ per cycle,

$72{ }^{\circ} \mathrm{C}$ for $\left.30 \mathrm{~s}\right\}$

11 cycles of $\left\{95^{\circ} \mathrm{C}\right.$ for $30 \mathrm{~s}, 55^{\circ} \mathrm{C}$ for $30 \mathrm{~s}, 72{ }^{\circ} \mathrm{C}$ for $\left.30 \mathrm{~s}\right\}$

$72{ }^{\circ} \mathrm{C}$ for $7 \mathrm{~min}$

$25^{\circ} \mathrm{C}$ until removed from thermocycler

Following PCR amplification, unincorporated primers and dNTPs were removed by adding $4 \mu \mathrm{l}$ of a Exo-SAP enzyme cocktail consisting of $1.4 \mu$ l Exonuclease I $(10 \mathrm{U} / \mu \mathrm{l})$ and $2.6 \mu \mathrm{l}(1 \mathrm{U} / \mu \mathrm{l})$ of shrimp alkaline phosphatase (SAP; USB Corp., Cleveland, OH) to each $10 \mu \mathrm{l}$ PCR reaction. Reactions were mixed briefly and incubated at $37{ }^{\circ} \mathrm{C}$ for $90 \mathrm{~min}$ and then $80^{\circ} \mathrm{C}$ for $20 \mathrm{~min}$ to inactivate the enzymes.

\section{Alelle specific primer extension (ASPE)}

ASPE reactions were also carried out in eleven 6-plexes and a single 4-plex. Multiplex primer extension reactions were conducted in a total volume of $10 \mu \mathrm{l}$ using $2.5 \mu \mathrm{l}$ of ABI Prism ${ }^{\circledR}$ SNaPshot ${ }^{\mathrm{TM}}$ multiplex kit mix (Applied Biosystems), $0.5 \mu \mathrm{l}$ of 10X AmpliTaq Gold ${ }^{\mathbb{R}}$ PCR buffer, $3 \mu 1$ of PCR template, $3 \mu \mathrm{l}$ of water, and $1 \mu \mathrm{l}$ of a stock solution of extension primers, which contained empirically balanced primers (approximately $1 \mu \mathrm{M}$ each). Extension reactions were incubated as follows: 25 cycles of $96{ }^{\circ} \mathrm{C}$ for $10 \mathrm{~s}$, $50{ }^{\circ} \mathrm{C}$ for $5 \mathrm{~s}$, and $60{ }^{\circ} \mathrm{C}$ for $30 \mathrm{~s}$. Excess fluorescentlylabeled ddNTPs were inactivated by addition of $1 \mu \mathrm{l}$ of SAP $(1 \mathrm{U} / \mu \mathrm{l})$. Reactions were mixed briefly and incubated at $37{ }^{\circ} \mathrm{C}$ for $40 \mathrm{~min}$ then $90{ }^{\circ} \mathrm{C}$ for $5 \mathrm{~min}$.

\section{Electrophoresis and typing}

A $1.0 \mu \mathrm{l}$ aliquot of each SAP-treated primer extension

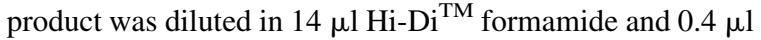
GS120-LIZ internal size standard (Applied Biosystems) and analyzed on the 16-capillary ABI Prism ${ }^{\circledR} 3100$ Genetic Analyzer (Applied Biosystems) using filter set E5 without prior denaturation of samples. Samples were injected electrokinetically for $13 \mathrm{~s}$ at $1 \mathrm{kV}$. Separations were performed in approximately $30 \mathrm{~min}$ on a $36 \mathrm{~cm}$ array using $\mathrm{POP}^{\mathrm{TM}}-6$ (Applied Biosystems). Automated allele calls were made in Genotyper ${ }^{\circledR} 3.7$ using an in-house macro based on fragment size and dye color.

\section{Analysis of data}

The data were analyzed with PowerMarker v3.07 [5]. Allele frequencies, expected heterozygosity values and pvalues (based on an exact test with 1000 reshufflings) for each marker are provided in Tables 2-4 for the three U.S. sample groups.

\section{Access to the data}

SNP marker information is available on the forensic SNP site: http://www.cstl.nist.gov/biotech/strbase/SNP.htm and genotyping results are posted at http://www.cstl.nist.gov/ biotech/strbase/NISTpop.htm.

\section{Results and discussion}

Tables 2-4 contain the observed allele frequencies for U.S. Caucasian, African-American, and Hispanic samples, respectively. The $\mathrm{C}$ and $\mathrm{T}$ allele frequencies were used to calculate the expected heterozygosities that were then compared to the observed frequencies of heterozygotes. A total of 10 of the 210 tests (70 loci $\times 3$ populations) for Hardy-Weinberg equilibrium indicated a deviation from the expected result. As has been noted before, it is reasonable to expect approximately $5 \%$, or 10 to 11 out of 210 , of 
Table 1

Information on 70 autosomal SNP loci examined in this study sorted by chromosome position. Locus numbers are arbitrary. Chromosome positions were determined using the July 2003 human genome reference sequence. GenBank and dbSNP information can be retrieved from http://www.ncbi.nlm.nih.gov. The SNP Consortium numbers (TSC \#) from http://snp.cshl.org are also listed

\begin{tabular}{|c|c|c|c|c|c|c|c|}
\hline $\begin{array}{l}\text { SNP } \\
\text { locus \# }\end{array}$ & Chromosome & $\begin{array}{l}\text { Chromosome } \\
\text { position }\end{array}$ & $\begin{array}{l}\text { GenBank } \\
\text { reference }\end{array}$ & $\begin{array}{l}\text { Reference } \\
\text { allele }\end{array}$ & $\begin{array}{l}\mathrm{dbSNP} \\
\text { reference }\end{array}$ & TSC \# & $\begin{array}{l}\text { PCR } \\
\text { Product size } \\
\end{array}$ \\
\hline 58 & 1 & $14,359,351$ & AL034395.6 & $\mathrm{T}$ & rs734664 & TSC0026510 & 64 \\
\hline 18 & 1 & $33,657,974$ & AL161643.2 & $\mathrm{T}$ & rs732889 & TSC0022461 & 65 \\
\hline 66 & 1 & $53,564,936$ & AL049745.9 & $\mathrm{T}$ & rs702490 & TSC0243017 & 73 \\
\hline 48 & 1 & $109,978,841$ & AL160006.1 & $\mathrm{C}$ & rs924181 & TSC0239374 & 63 \\
\hline 24 & 1 & $164,087,184$ & AL009182.1 & $\mathrm{T}$ & rs2013526 & TSC0013419 & 65 \\
\hline 17 & 1 & $181,421,773$ & AC009481.4 & $\mathrm{C}$ & rs997568 & TSC0002990 & 64 \\
\hline 50 & 2 & $7,855,560$ & AC092580.3 & $\mathrm{C}$ & rs772436 & TSC0013222 & 69 \\
\hline 35 & 2 & $12,145,530$ & AC018866 & $\mathrm{T}$ & rs896499 & TSC0170191 & 78 \\
\hline 22 & 2 & $33,160,796$ & AL133244.1 & $\mathrm{C}$ & rs1020636 & TSC0224883 & 61 \\
\hline 28 & 2 & $53,012,403$ & AC018713.7 & $\mathrm{C}$ & rs2015632 & TSC0006100 & 70 \\
\hline 30 & 2 & $59,987,336$ & AC007100.3 & $\mathrm{C}$ & rs1019264 & TSC0222995 & 80 \\
\hline 38 & 2 & $155,136,334$ & AC008166.2 & $\mathrm{T}$ & rs1079861 & TSC0066071 & 78 \\
\hline 52 & 2 & $205,040,634$ & AC009965.9 & $\mathrm{T}$ & rs3096741 & TSC0211925 & 64 \\
\hline 1 & 2 & $221,198,958$ & AC008064 & $\mathrm{T}$ & rs734295 & TSC0025620 & 62 \\
\hline 13 & 4 & $182,888,181$ & AC019235.6 & $\mathrm{T}$ & rs716360 & TSC0005559 & 62 \\
\hline 19 & 5 & $118,134,847$ & AC008444.4 & $\mathrm{T}$ & rs730907 & TSC0018534 & 62 \\
\hline 16 & 5 & $132,731,841$ & AC010307.7 & $\mathrm{T}$ & rs733023 & TSC0022796 & 61 \\
\hline 67 & 5 & $153,889,557$ & AC026688.7 & $\mathrm{C}$ & rs880083 & TSC0214907 & 73 \\
\hline 14 & 5 & $164,661,137$ & AC008644 & $\mathrm{C}$ & rs1024997 & TSC0248350 & 89 \\
\hline 26 & 6 & $3,295,184$ & AL160398.2 & $\mathrm{T}$ & rs730488 & TSC0017645 & 68 \\
\hline 54 & 6 & $15,118,209$ & AL050335.3 & $\mathrm{C}$ & rs927628 & TSC0244208 & 70 \\
\hline 44 & 6 & $39,929,605$ & AL136089.9 & $\mathrm{T}$ & rs716856 & TSC0009879 & 62 \\
\hline 10 & 6 & $65,000,740$ & AL078597.1 & $\mathrm{C}$ & rs1028484 & TSC0253002 & 68 \\
\hline 3 & 6 & $88,362,198$ & AL133211.9 & $\mathrm{T}$ & rs1075665 & TSC0021586 & 63 \\
\hline 4 & 6 & $119,778,606$ & AL360215.1 & $\mathrm{C}$ & rs924397 & TSC0239700 & 63 \\
\hline 45 & 6 & $124,123,520$ & AL354936.2 & $\mathrm{T}$ & rs765533 & TSC0069344 & 77 \\
\hline 56 & 6 & $166,939,350$ & Z98049.1 & $\mathrm{C}$ & rs916388 & TSC0201398 & 61 \\
\hline 41 & 7 & $15,262,947$ & AC012061.4 & $\mathrm{T}$ & rs1072292 & TSC0010262 & 79 \\
\hline 8 & 7 & $51,706,494$ & AC022458.3 & $\mathrm{T}$ & rs997556 & TSC0002961 & 61 \\
\hline 7 & 7 & $102,804,023$ & AC006316.2 & $\mathrm{T}$ & rs123714 & TSC0105900 & 64 \\
\hline 33 & 8 & $57,612,000$ & AC009597.5 & $\mathrm{T}$ & rs919023 & TSC0232293 & 81 \\
\hline 49 & 8 & $63,755,655$ & AC018398 & $\mathrm{C}$ & rs734701 & TSC0026586 & 69 \\
\hline 37 & 8 & $103,506,597$ & AP002852.3 & $\mathrm{C}$ & rs892503 & TSC0163923 & 76 \\
\hline 39 & 8 & $117,079,183$ & AF130343.1 & $\mathrm{T}$ & rs6469629 & TSC0218207 & 69 \\
\hline 32 & 9 & $17,592,496$ & AL133214.1 & $\mathrm{T}$ & rs1008730 & TSC0087190 & 69 \\
\hline 51 & 10 & $27,923,937$ & AC024606.1 & $\mathrm{T}$ & rs997750 & TSC0003290 & 63 \\
\hline 12 & 10 & $82,436,151$ & AC013242.8 & $\mathrm{T}$ & rs922992 & TSC0237737 & 63 \\
\hline 43 & 10 & $113,292,473$ & AL136119.1 & $\mathrm{T}$ & rs585070 & TSC0231103 & 81 \\
\hline 20 & 11 & $19,942,027$ & AC079361.1 & $\mathrm{C}$ & rs729999 & TSC0016655 & 60 \\
\hline 63 & 11 & $35,506,187$ & AL354921.1 & $\mathrm{T}$ & rs627119 & TSC0016429 & 59 \\
\hline 46 & 11 & $103,380,259$ & AP003043.2 & $\mathrm{C}$ & rs1021290 & TSC0225784 & 80 \\
\hline 62 & 11 & $131,628,725$ & AP004248.2 & $\mathrm{C}$ & rs921269 & TSC0235383 & 76 \\
\hline 40 & 12 & $30,160,004$ & AC068811.8 & $\mathrm{T}$ & rs959566 & TSC0261969 & 75 \\
\hline 60 & 12 & $100,652,449$ & AC063950.3 & $\mathrm{T}$ & rs730013 & TSC0016681 & 72 \\
\hline 36 & 13 & $37,899,740$ & AL158194.1 & $\mathrm{C}$ & rs730249 & TSC0017130 & 65 \\
\hline 2 & 13 & $68,098,515$ & AL162378.1 & $\mathrm{C}$ & rs2018205 & TSC0062893 & 108 \\
\hline 59 & 13 & $98,078,430$ & AL445184.1 & $\mathrm{T}$ & rs1105576 & TSC0135614 & 67 \\
\hline 69 & 13 & $107,113,189$ & AL136132.1 & $\mathrm{C}$ & rs729549 & TSC0015745 & 72 \\
\hline 23 & 14 & $53,115,754$ & AL133444.4 & $\mathrm{T}$ & rs911621 & TSC0193791 & 60 \\
\hline 57 & 14 & $82,658,064$ & AL583743.3 & $\mathrm{C}$ & rs734656 & TSC0026489 & 64 \\
\hline 5 & 15 & $20,547,981$ & AC016204.1 & $\mathrm{T}$ & rs999842 & TSC0014520 & 64 \\
\hline 27 & 15 & $52,239,965$ & AC022302.7 & $\mathrm{T}$ & rs719211 & TSC0043836 & 82 \\
\hline 70 & 15 & $58,792,647$ & AC022898.1 & $\mathrm{T}$ & rs 877228 & TSC0209754 & 62 \\
\hline 25 & 17 & $32,063,659$ & AC011824.8 & $\mathrm{C}$ & rs727206 & TSC0061444 & 68 \\
\hline
\end{tabular}


Table 1 (Continued)

\begin{tabular}{|c|c|c|c|c|c|c|c|}
\hline $\begin{array}{l}\text { SNP } \\
\text { locus \# }\end{array}$ & Chromosome & $\begin{array}{l}\text { Chromosome } \\
\text { position }\end{array}$ & $\begin{array}{l}\text { GenBank } \\
\text { reference }\end{array}$ & $\begin{array}{l}\text { Reference } \\
\text { allele }\end{array}$ & $\begin{array}{l}\mathrm{dbSNP} \\
\text { reference }\end{array}$ & TSC \# & $\begin{array}{l}\text { PCR } \\
\text { Product size }\end{array}$ \\
\hline 21 & 17 & $39,523,608$ & AC007455.7 & $\mathrm{C}$ & rs2010209 & TSC0005110 & 70 \\
\hline 6 & 17 & $55,624,843$ & AC007114.8 & $\mathrm{C}$ & rs917927 & TSC0230724 & 74 \\
\hline 42 & 17 & $79,577,854$ & AC127496.5 & $\mathrm{C}$ & rs868432 & TSC0124845 & 70 \\
\hline 9 & 18 & $22,615,097$ & AC018371.1 & $\mathrm{T}$ & rs1017415 & TSC0220510 & 70 \\
\hline 47 & 18 & $32,376,937$ & AC131053.2 & $\mathrm{T}$ & rs4105107 & TSC0133660 & 71 \\
\hline 65 & 19 & $1,126,396$ & AC120982.2 & $\mathrm{T}$ & rs873289 & TSC0202573 & 65 \\
\hline 53 & 19 & $16,310,517$ & AC020917.4 & $\mathrm{C}$ & rs 1000329 & TSC0015380 & 67 \\
\hline 64 & 20 & $23,525,035$ & AL121894.2 & $\mathrm{C}$ & rs1003204 & TSC0038789 & 68 \\
\hline 55 & 20 & $25,048,105$ & AL080312.1 & $\mathrm{C}$ & rs743018 & TSC0113727 & 71 \\
\hline 68 & 20 & $43,388,976$ & AL034419.2 & $\mathrm{T}$ & rs4467339 & TSC0215392 & 66 \\
\hline 29 & 20 & $60,743,636$ & AL160412.1 & $\mathrm{T}$ & rs1000322 & TSC0015372 & 75 \\
\hline 15 & 21 & $17,486,896$ & AP001669 & $\mathrm{T}$ & rs 18579 & TSC0003543 & 67 \\
\hline 31 & 22 & $34,220,248$ & AL022334.1 & $\mathrm{C}$ & rs736210 & TSC0029823 & 62 \\
\hline 61 & 22 & $35,362,839$ & AL049749.2 & $\mathrm{C}$ & rs738518 & TSC0112989 & 64 \\
\hline 11 & 22 & $41,403,208$ & AL049757.1 & $\mathrm{C}$ & rs738532 & TSC0113010 & 71 \\
\hline 34 & 22 & $41,810,649$ & Z82214.2 & $\mathrm{T}$ & rs 138952 & TSC0117592 & 64 \\
\hline
\end{tabular}

Table 2

Allele frequencies observed for 74 U.S. Caucasians listed by SNP locus number (see Table 1)

\begin{tabular}{|c|c|c|c|c|c|c|c|c|c|c|c|c|}
\hline \multicolumn{13}{|c|}{ Caucasian $(N=74)$} \\
\hline & 1 & 2 & 3 & 4 & 5 & 6 & 7 & 8 & 9 & 10 & 11 & 12 \\
\hline $\mathrm{CC}$ & 0.243 & 0.405 & 0.068 & 0.581 & 0.311 & 0.149 & 0.486 & 0.108 & 0.203 & 0.068 & 0.257 & 0.054 \\
\hline $\mathrm{TT}$ & 0.243 & 0.135 & 0.514 & 0.135 & 0.189 & 0.338 & 0.122 & 0.378 & 0.284 & 0.459 & 0.216 & 0.365 \\
\hline CT & 0.514 & 0.459 & 0.419 & 0.284 & 0.500 & 0.514 & 0.392 & 0.514 & 0.514 & 0.473 & 0.527 & 0.581 \\
\hline $\mathrm{He}$ & 0.500 & 0.463 & 0.401 & 0.401 & 0.493 & 0.482 & 0.433 & 0.463 & 0.497 & 0.423 & 0.499 & 0.452 \\
\hline \multirow[t]{2}{*}{$P$} & 0.816 & 1.000 & 1.000 & 0.008 & 0.816 & 0.475 & 0.413 & 0.305 & 1.000 & 0.269 & 0.818 & 0.021 \\
\hline & 13 & 14 & 15 & 16 & 17 & 18 & 19 & 20 & 21 & 22 & 23 & 24 \\
\hline $\mathrm{CC}$ & 0.068 & 0.243 & 0.392 & 0.446 & 0.243 & 0.162 & 0.473 & 0.365 & 0.270 & 0.108 & 0.270 & 0.203 \\
\hline $\mathrm{TT}$ & 0.514 & 0.311 & 0.122 & 0.149 & 0.284 & 0.324 & 0.054 & 0.203 & 0.189 & 0.432 & 0.176 & 0.270 \\
\hline CT & 0.419 & 0.446 & 0.486 & 0.405 & 0.473 & 0.514 & 0.473 & 0.432 & 0.541 & 0.459 & 0.554 & 0.527 \\
\hline $\mathrm{He}$ & 0.401 & 0.498 & 0.463 & 0.456 & 0.499 & 0.487 & 0.412 & 0.487 & 0.497 & 0.447 & 0.496 & 0.498 \\
\hline \multirow[t]{2}{*}{$P$} & 1.000 & 0.220 & 0.805 & 0.183 & 0.663 & 0.818 & 0.163 & 0.348 & 0.485 & 1.000 & 0.362 & 0.650 \\
\hline & 25 & 26 & 27 & 28 & 29 & 30 & 31 & 32 & 33 & 34 & 35 & 36 \\
\hline $\mathrm{CC}$ & 0.243 & 0.176 & 0.162 & 0.068 & 0.257 & 0.432 & 0.419 & 0.527 & 0.122 & 0.581 & 0.257 & 0.108 \\
\hline $\mathrm{TT}$ & 0.203 & 0.446 & 0.432 & 0.689 & 0.284 & 0.149 & 0.122 & 0.122 & 0.311 & 0.068 & 0.203 & 0.243 \\
\hline CT & 0.554 & 0.378 & 0.405 & 0.243 & 0.459 & 0.419 & 0.459 & 0.351 & 0.568 & 0.351 & 0.541 & 0.649 \\
\hline $\mathrm{He}$ & 0.499 & 0.463 & 0.463 & 0.307 & 0.500 & 0.460 & 0.456 & 0.418 & 0.482 & 0.368 & 0.499 & 0.491 \\
\hline \multirow[t]{2}{*}{$P$} & 0.480 & 0.135 & 0.327 & 0.119 & 0.350 & 0.302 & 0.797 & 0.092 & 0.088 & 0.538 & 0.642 & 0.008 \\
\hline & 37 & 38 & 39 & 40 & 41 & 42 & 43 & 44 & 45 & 46 & 47 & 48 \\
\hline $\mathrm{CC}$ & 0.378 & 0.095 & 0.378 & 0.149 & 0.297 & 0.311 & 0.257 & 0.473 & 0.122 & 0.189 & 0.162 & 0.351 \\
\hline $\mathrm{TT}$ & 0.122 & 0.473 & 0.149 & 0.514 & 0.216 & 0.149 & 0.216 & 0.095 & 0.446 & 0.284 & 0.351 & 0.162 \\
\hline $\mathrm{CT}$ & 0.500 & 0.432 & 0.473 & 0.338 & 0.486 & 0.541 & 0.527 & 0.432 & 0.432 & 0.527 & 0.486 & 0.486 \\
\hline $\mathrm{He}$ & 0.467 & 0.428 & 0.474 & 0.433 & 0.497 & 0.487 & 0.499 & 0.428 & 0.447 & 0.496 & 0.482 & 0.482 \\
\hline \multirow[t]{2}{*}{$P$} & 0.444 & 0.790 & 0.806 & 0.060 & 0.827 & 0.491 & 0.822 & 0.791 & 0.790 & 0.635 & 0.803 & 0.802 \\
\hline & 49 & 50 & 51 & 52 & 53 & 54 & 55 & 56 & 57 & 58 & 59 & 60 \\
\hline $\mathrm{CC}$ & 0.135 & 0.203 & 0.176 & 0.365 & 0.095 & 0.284 & 0.446 & 0.419 & 0.149 & 0.081 & 0.081 & 0.351 \\
\hline $\mathrm{TT}$ & 0.568 & 0.378 & 0.257 & 0.176 & 0.541 & 0.189 & 0.135 & 0.108 & 0.392 & 0.662 & 0.527 & 0.203 \\
\hline $\mathrm{CT}$ & 0.297 & 0.419 & 0.568 & 0.459 & 0.365 & 0.527 & 0.419 & 0.473 & 0.459 & 0.257 & 0.392 & 0.446 \\
\hline $\mathrm{He}$ & 0.407 & 0.485 & 0.497 & 0.482 & 0.401 & 0.496 & 0.452 & 0.452 & 0.470 & 0.331 & 0.401 & 0.489 \\
\hline$P$ & 0.008 & 0.229 & 0.244 & 0.638 & 0.566 & 0.645 & 0.472 & 0.612 & 0.618 & 0.068 & 0.785 & 0.331 \\
\hline
\end{tabular}


Table 2 (Continued)

\begin{tabular}{lllllllllll}
\hline \multicolumn{1}{l}{ Caucasian $(N=74)$} & \multicolumn{10}{l}{0} \\
\hline & 61 & 62 & 63 & 64 & 65 & 66 & 67 & 68 & 69 & 70 \\
CC & 0.068 & 0.473 & 0.189 & 0.162 & 0.243 & 0.378 & 0.486 & 0.324 & 0.216 & 0.284 \\
TT & 0.608 & 0.027 & 0.486 & 0.284 & 0.216 & 0.162 & 0.054 & 0.108 & 0.351 & 0.149 \\
CT & 0.324 & 0.500 & 0.324 & 0.554 & 0.541 & 0.459 & 0.459 & 0.568 & 0.432 & 0.568 \\
He & 0.354 & 0.401 & 0.456 & 0.493 & 0.500 & 0.477 & 0.407 & 0.477 & 0.491 & 0.491 \\
$P$ & 0.326 & $\mathbf{0 . 0 4 3}$ & $\mathbf{0 . 0 1 1}$ & 0.232 & 0.493 & 0.805 & 0.401 & 0.142 & 0.346 & 0.263 \\
\hline
\end{tabular}

$\mathrm{He}=$ expected heterozygosity; $P$ : Fisher's exact test for Hardy-Weinberg equilibrium, based on 1000 shufflings. Values that are below the minimum allele frequency of $5 / 2 \mathrm{~N}(0.034)$ are italicized.

Table 3

Allele frequencies observed for 71 African-Americans listed by SNP locus number

\begin{tabular}{|c|c|c|c|c|c|c|c|c|c|c|c|c|}
\hline \multicolumn{13}{|c|}{ African-American $(N=71)$} \\
\hline & 1 & 2 & 3 & 4 & 5 & 6 & 7 & 8 & 9 & 10 & 11 & 12 \\
\hline $\mathrm{CC}$ & 0.648 & 0.113 & 0.141 & 0.352 & 0.141 & 0.127 & 0.648 & 0.183 & 0.225 & 0.014 & 0.070 & 0.394 \\
\hline TT & 0.070 & 0.352 & 0.437 & 0.141 & 0.338 & 0.563 & 0.070 & 0.408 & 0.338 & 0.662 & 0.549 & 0.155 \\
\hline $\mathrm{CT}$ & 0.282 & 0.535 & 0.423 & 0.507 & 0.521 & 0.310 & 0.282 & 0.408 & 0.437 & 0.324 & 0.380 & 0.451 \\
\hline $\mathrm{He}$ & 0.333 & 0.471 & 0.456 & 0.478 & 0.481 & 0.405 & 0.333 & 0.475 & 0.494 & 0.290 & 0.385 & 0.471 \\
\hline \multirow[t]{2}{*}{$P$} & 0.278 & 0.207 & 0.441 & 0.808 & 0.468 & 0.043 & 0.275 & 0.226 & 0.225 & 0.680 & 0.760 & 0.624 \\
\hline & 13 & 14 & 15 & 16 & 17 & 18 & 19 & 20 & 21 & 22 & 23 & 24 \\
\hline $\mathrm{CC}$ & 0.141 & 0.296 & 0.239 & 0.479 & 0.113 & 0.113 & 0.634 & 0.197 & 0.070 & 0.028 & 0.282 & 0.268 \\
\hline $\mathrm{TT}$ & 0.451 & 0.113 & 0.338 & 0.085 & 0.479 & 0.451 & 0.042 & 0.366 & 0.606 & 0.648 & 0.239 & 0.239 \\
\hline CT & 0.408 & 0.592 & 0.423 & 0.437 & 0.408 & 0.437 & 0.324 & 0.437 & 0.324 & 0.324 & 0.479 & 0.493 \\
\hline $\mathrm{He}$ & 0.452 & 0.483 & 0.495 & 0.422 & 0.433 & 0.443 & 0.325 & 0.486 & 0.357 & 0.308 & 0.499 & 0.500 \\
\hline \multirow[t]{2}{*}{$P$} & 0.298 & 0.093 & 0.158 & 0.784 & 0.396 & 1.000 & 0.720 & 0.469 & 0.326 & 1.000 & 0.644 & 1.000 \\
\hline & 25 & 26 & 27 & 28 & 29 & 30 & 31 & 32 & 33 & 34 & 35 & 36 \\
\hline $\mathrm{CC}$ & 0.099 & 0.394 & 0.239 & 0.225 & 0.113 & 0.352 & 0.380 & 0.113 & 0.197 & 0.493 & 0.113 & 0.352 \\
\hline $\mathrm{TT}$ & 0.394 & 0.155 & 0.254 & 0.282 & 0.451 & 0.169 & 0.183 & 0.423 & 0.338 & 0.042 & 0.535 & 0.197 \\
\hline $\mathrm{CT}$ & 0.507 & 0.451 & 0.507 & 0.493 & 0.437 & 0.479 & 0.437 & 0.465 & 0.465 & 0.465 & 0.352 & 0.451 \\
\hline $\mathrm{He}$ & 0.456 & 0.471 & 0.500 & 0.498 & 0.443 & 0.483 & 0.481 & 0.452 & 0.490 & 0.398 & 0.411 & 0.488 \\
\hline \multirow[t]{2}{*}{$P$} & 0.310 & 0.606 & 1.000 & 1.000 & 1.000 & 0.805 & 0.315 & 0.799 & 0.645 & 0.234 & 0.157 & 0.622 \\
\hline & 37 & 38 & 39 & 40 & 41 & 42 & 43 & 44 & 45 & 46 & 47 & 48 \\
\hline $\mathrm{CC}$ & 0.324 & 0.211 & 0.113 & 0.141 & 0.113 & 0.225 & 0.056 & 0.606 & 0.380 & 0.211 & 0.099 & 0.380 \\
\hline $\mathrm{TT}$ & 0.183 & 0.366 & 0.592 & 0.465 & 0.549 & 0.310 & 0.479 & 0.056 & 0.183 & 0.366 & 0.465 & 0.169 \\
\hline $\mathrm{CT}$ & 0.493 & 0.423 & 0.296 & 0.394 & 0.338 & 0.465 & 0.465 & 0.338 & 0.437 & 0.423 & 0.437 & 0.451 \\
\hline $\mathrm{He}$ & 0.490 & 0.488 & 0.385 & 0.448 & 0.405 & 0.496 & 0.411 & 0.349 & 0.481 & 0.488 & 0.433 & 0.478 \\
\hline \multirow[t]{2}{*}{$P$} & 1.000 & 0.335 & 0.061 & 0.182 & 0.159 & 0.639 & 0.256 & 0.488 & 0.331 & 0.336 & 0.786 & 0.625 \\
\hline & 49 & 50 & 51 & 52 & 53 & 54 & 55 & 56 & 57 & 58 & 59 & 60 \\
\hline $\mathrm{CC}$ & 0.085 & 0.465 & 0.310 & 0.099 & 0.423 & 0.282 & 0.211 & 0.408 & 0.042 & 0.239 & 0.282 & 0.352 \\
\hline $\mathrm{TT}$ & 0.549 & 0.070 & 0.099 & 0.648 & 0.169 & 0.141 & 0.310 & 0.141 & 0.535 & 0.225 & 0.183 & 0.211 \\
\hline $\mathrm{CT}$ & 0.366 & 0.465 & 0.592 & 0.254 & 0.408 & 0.577 & 0.479 & 0.451 & 0.423 & 0.535 & 0.535 & 0.437 \\
\hline $\mathrm{He}$ & 0.392 & 0.422 & 0.478 & 0.349 & 0.468 & 0.490 & 0.495 & 0.464 & 0.378 & 0.500 & 0.495 & 0.490 \\
\hline \multirow[t]{2}{*}{$P$} & 0.369 & 0.401 & 0.081 & 0.030 & 0.307 & 0.221 & 0.626 & 0.616 & 0.532 & 0.633 & 0.475 & 0.337 \\
\hline & 61 & 62 & 63 & 64 & 65 & 66 & 67 & 68 & 69 & 70 & & \\
\hline $\mathrm{CC}$ & 0.310 & 0.352 & 0.268 & 0.479 & 0.183 & 0.423 & 0.592 & 0.380 & 0.338 & 0.296 & & \\
\hline $\mathrm{TT}$ & 0.225 & 0.141 & 0.183 & 0.099 & 0.310 & 0.169 & 0.113 & 0.113 & 0.197 & 0.141 & & \\
\hline $\mathrm{CT}$ & 0.465 & 0.507 & 0.549 & 0.423 & 0.507 & 0.408 & 0.296 & 0.507 & 0.465 & 0.563 & & \\
\hline $\mathrm{He}$ & 0.496 & 0.478 & 0.496 & 0.428 & 0.492 & 0.468 & 0.385 & 0.464 & 0.490 & 0.488 & & \\
\hline$P$ & 0.634 & 0.803 & 0.469 & 0.786 & 1.000 & 0.296 & 0.059 & 0.459 & 0.626 & 0.226 & & \\
\hline
\end{tabular}

See Table $1 . \mathrm{He}=$ expected heterozygosity; $P$ : Fisher's exact test for Hardy-Weinberg equilibrium, based on 1000 shufflings. Values that are below the minimum allele frequency of $5 / 2 \mathrm{~N}(0.035)$ are highlighted. 
Table 4

Allele frequencies observed for 44 U.S. Hispanics listed by SNP locus number (see Table 1)

\begin{tabular}{|c|c|c|c|c|c|c|c|c|c|c|c|c|}
\hline \multicolumn{13}{|c|}{ Hispanic $(N=44)$} \\
\hline & 1 & 2 & 3 & 4 & 5 & 6 & 7 & 8 & 9 & 10 & 11 & 12 \\
\hline $\mathrm{CC}$ & 0.455 & 0.477 & 0.114 & 0.364 & 0.364 & 0.045 & 0.432 & 0.182 & 0.227 & 0.091 & 0.182 & 0.205 \\
\hline $\mathrm{TT}$ & 0.068 & 0.045 & 0.341 & 0.136 & 0.136 & 0.432 & 0.114 & 0.386 & 0.273 & 0.409 & 0.341 & 0.341 \\
\hline $\mathrm{CT}$ & 0.477 & 0.477 & 0.545 & 0.500 & 0.500 & 0.523 & 0.455 & 0.432 & 0.500 & 0.500 & 0.477 & 0.455 \\
\hline $\mathrm{He}$ & 0.425 & 0.407 & 0.474 & 0.474 & 0.474 & 0.425 & 0.449 & 0.479 & 0.499 & 0.449 & 0.487 & 0.491 \\
\hline \multirow[t]{2}{*}{$P$} & 0.723 & 0.441 & 0.522 & 1.000 & 1.000 & 0.177 & 1.000 & 0.545 & 1.000 & 0.741 & 1.000 & 0.724 \\
\hline & 13 & 14 & 15 & 16 & 17 & 18 & 19 & 20 & 21 & 22 & 23 & 24 \\
\hline $\mathrm{CC}$ & 0.068 & 0.341 & 0.500 & 0.386 & 0.114 & 0.295 & 0.477 & 0.273 & 0.136 & 0.091 & 0.250 & 0.273 \\
\hline $\mathrm{TT}$ & 0.568 & 0.205 & 0.091 & 0.159 & 0.545 & 0.318 & 0.068 & 0.205 & 0.364 & 0.455 & 0.364 & 0.273 \\
\hline CT & 0.364 & 0.455 & 0.409 & 0.455 & 0.341 & 0.386 & 0.455 & 0.523 & 0.500 & 0.455 & 0.386 & 0.455 \\
\hline $\mathrm{He}$ & 0.375 & 0.491 & 0.416 & 0.474 & 0.407 & 0.500 & 0.416 & 0.498 & 0.474 & 0.434 & 0.494 & 0.500 \\
\hline \multirow[t]{2}{*}{$P$} & 1.000 & 0.777 & 1.000 & 0.782 & 0.311 & 0.135 & 0.719 & 1.000 & 1.000 & 1.000 & 0.226 & 0.565 \\
\hline & 25 & 26 & 27 & 28 & 29 & 30 & 31 & 32 & 33 & 34 & 35 & 36 \\
\hline $\mathrm{CC}$ & 0.318 & 0.205 & 0.227 & 0.091 & 0.227 & 0.114 & 0.523 & 0.477 & 0.136 & 0.568 & 0.182 & 0.318 \\
\hline $\mathrm{TT}$ & 0.114 & 0.455 & 0.227 & 0.682 & 0.364 & 0.295 & 0.182 & 0.091 & 0.432 & 0.091 & 0.318 & 0.227 \\
\hline CT & 0.568 & 0.341 & 0.545 & 0.227 & 0.409 & 0.591 & 0.295 & 0.432 & 0.432 & 0.341 & 0.500 & 0.455 \\
\hline $\mathrm{He}$ & 0.479 & 0.469 & 0.500 & 0.325 & 0.491 & 0.483 & 0.442 & 0.425 & 0.456 & 0.386 & 0.491 & 0.496 \\
\hline \multirow[t]{2}{*}{$P$} & 0.326 & 0.097 & 0.766 & 0.063 & 0.365 & 0.198 & 0.036 & 1.000 & 0.752 & 0.435 & 1.000 & 0.558 \\
\hline & 37 & 38 & 39 & 40 & 41 & 42 & 43 & 44 & 45 & 46 & 47 & 48 \\
\hline $\mathrm{CC}$ & 0.523 & 0.045 & 0.455 & 0.114 & 0.295 & 0.250 & 0.205 & 0.523 & 0.091 & 0.136 & 0.205 & 0.477 \\
\hline $\mathrm{TT}$ & 0.045 & 0.568 & 0.091 & 0.477 & 0.159 & 0.136 & 0.432 & 0.023 & 0.455 & 0.318 & 0.477 & 0.023 \\
\hline $\mathrm{CT}$ & 0.432 & 0.386 & 0.455 & 0.409 & 0.545 & 0.614 & 0.364 & 0.455 & 0.455 & 0.545 & 0.318 & 0.500 \\
\hline $\mathrm{He}$ & 0.386 & 0.363 & 0.434 & 0.434 & 0.491 & 0.494 & 0.474 & 0.375 & 0.434 & 0.483 & 0.463 & 0.397 \\
\hline \multirow[t]{2}{*}{$P$} & 0.694 & 1.000 & 1.000 & 0.727 & 0.542 & 0.135 & 0.119 & 0.248 & 1.000 & 0.522 & 0.048 & 0.143 \\
\hline & 49 & 50 & 51 & 52 & 53 & 54 & 55 & 56 & 57 & 58 & 59 & 60 \\
\hline $\mathrm{CC}$ & 0.068 & 0.318 & 0.227 & 0.29545 & 0.159 & 0.250 & 0.409 & 0.500 & 0.182 & 0.023 & 0.136 & 0.182 \\
\hline $\mathrm{TT}$ & 0.636 & 0.227 & 0.318 & 0.25 & 0.341 & 0.295 & 0.136 & 0.068 & 0.455 & 0.636 & 0.500 & 0.250 \\
\hline $\mathrm{CT}$ & 0.295 & 0.455 & 0.455 & 0.45455 & 0.500 & 0.455 & 0.455 & 0.432 & 0.364 & 0.341 & 0.364 & 0.568 \\
\hline $\mathrm{He}$ & 0.339 & 0.496 & 0.496 & 0.499 & 0.483 & 0.499 & 0.463 & 0.407 & 0.463 & 0.312 & 0.434 & 0.498 \\
\hline \multirow[t]{2}{*}{$P$} & 0.381 & 0.562 & 0.563 & 0.576 & 1.000 & 0.558 & 1.000 & 1.000 & 0.168 & 1.000 & 0.294 & 0.564 \\
\hline & 61 & 62 & 63 & 64 & 65 & 66 & 67 & 68 & 69 & 70 & & \\
\hline $\mathrm{CC}$ & 0.068 & 0.409 & 0.205 & 0.205 & 0.386 & 0.432 & 0.295 & 0.295 & 0.273 & 0.318 & & \\
\hline $\mathrm{TT}$ & 0.455 & 0.091 & 0.341 & 0.205 & 0.227 & 0.182 & 0.227 & 0.227 & 0.227 & 0.205 & & \\
\hline $\mathrm{CT}$ & 0.477 & 0.500 & 0.455 & 0.591 & 0.386 & 0.386 & 0.477 & 0.477 & 0.500 & 0.477 & & \\
\hline $\mathrm{He}$ & 0.425 & 0.449 & 0.491 & 0.500 & 0.487 & 0.469 & 0.498 & 0.498 & 0.499 & 0.494 & & \\
\hline$P$ & 0.721 & 0.744 & 0.777 & 0.383 & 0.191 & 0.336 & 0.797 & 0.754 & 1.000 & 1.000 & & \\
\hline
\end{tabular}

$\mathrm{He}=$ expected heterozygosity; $P$ : Fisher's exact test for Hardy-Weinberg equilibrium, based on 1000 shufflings. Values that are below the minimum allele frequency of $5 / 2 \mathrm{~N}(0.057)$ are highlighted.

the comparisons to deviate from Hardy-Weinberg equilibrium (see $[6,7]$ ). Those $P$-values significant at the $95 \%$ confidence level are those less than 0.05 and bolded in Tables 2-4. Six were observed in Caucasian samples and two in both the African-American and Hispanic data sets.

Typically the minimum number of samples needed to provide a robust estimate for allele frequencies with loci containing 5-15 alleles is 100-150 samples for each population [8]. Since we are measuring bi-allelic markers in this study that only have three possible genotypes (CC, TT or CT), a smaller number of samples should be sufficient [8] provided that we utilize a minimum allele frequency, such as
$5 / 2 \mathrm{~N}$ [9]. An examination of the data in Tables 2-4 finds a total of 10 allele frequency measurements (out of 630 total) below the $5 / 2 \mathrm{~N}$ threshold across the three populations. Thus, to be conservative a minimum allele frequency of $0.034,0.035$, and 0.057 should be used with those infrequently observed alleles in our Caucasian (Table 2), AfricanAmerican (Table 3), and Hispanic (Table 4) population data sets, respectively.

In order to evaluate the minimum number of SNP loci needed to distinguish all 189 samples from one another, we ranked the loci according to their levels of observed heterozygosity and $P$-values obtained upon testing for Hardy-Weinberg equilibrium. The top 12 loci according 
Table 5

Number of unique genotypes observed in the set of 189 unrelated individuals examined in this study with the addition of each SNP from a set of the top 12 SNPs ranked by observed heterozygosity and $P$-value. Locus number correlates to Table 1

\begin{tabular}{lcc}
\hline $\begin{array}{l}\text { No. of } \\
\text { markers } \\
\text { combined }\end{array}$ & $\begin{array}{l}\text { Locus \# } \\
\text { (see Table 1) }\end{array}$ & $\begin{array}{l}\text { No. of } \\
\text { unique } \\
\text { genotypes }\end{array}$ \\
\hline 1 & 5 & 3 \\
2 & 24 & 9 \\
3 & 2 & 27 \\
4 & 62 & 64 \\
5 & 48 & 107 \\
6 & 70 & 145 \\
7 & 54 & 160 \\
8 & 12 & 175 \\
9 & 68 & 182 \\
10 & 42 & 186 \\
11 & 36 & 188 \\
12 & 25 & 189 \\
\hline
\end{tabular}

to these ranking criteria are listed in Table 5 along with the number of unique genotypes observed when combining subsequent SNP markers. The 12 selected SNPs possessed an observed heterozygosity of $>0.45$ in all three populations examined and thus would be expected to exhibit more differences between samples. All of the 189 samples in this study were individualized with these 12 SNP loci (Table 5). However, it is likely that the addition of more than 12 SNP loci will be required to resolve larger sets of unrelated individuals from one another. By way of comparison, in these same 189 individuals all but one pair is resolved from one another with three of the STR loci possessing the highest heterozygosity values (D2S1338, D18S51, and FGA) run with the Identifiler kit. The final pair of unrelated samples could be resolved with the combination of 4 STR loci: D2S1338, D18S51, FGA, and VWA (data not shown).

While not as polymorphic as multiallelic STRs, it appears that biallelic SNPs are still able to separate unrelated and related individuals from one another with a reasonable number of loci. However, the construction of large robust multiplexes, such as demonstrated by Sanchez et al. [10], with these and other SNPs will be necessary to enable routine recovery of genetic data from degraded and low copy number DNA present in forensic evidence [11]. The small PCR product sizes from these particular autosomal SNPs (see Table 1) have enabled recovery of information from severely degraded DNA samples and assisted in the identification of some of the World Trade Center victims with data generated by Orchid Cellmark (personal communication, Bob Shaler, Office of the Chief Medical Examiner, New York City).

This paper follows the guidelines for publication of population data requested by the journal [12].

\section{Acknowledgements}

This work was funded by the National Institute of Justice (NIJ) through an interagency agreement with the NIST Office of Law Enforcement Standards. Jeanine Baisch and Bob Giles from Orchid Cellmark (Dallas, TX) enabled this work with these 70 markers through providing their PCR primer information. The first tests with these SNP markers were done as part of a concordance study with Orchid Cellmark at the request of NIJ's World Trade Center Kinship and Data Analysis Panel in July 2002. Gordon Spangler, a graduate student from American University, helped with some of the early tests. Initial preparation of the population samples by Margaret Kline, Jan Redman, and Richard Schoske is gratefully acknowledged. Certain commercial equipment, instruments and materials are identified in order to specify experimental procedures as completely as possible. In no case does such identification imply a recommendation or endorsement by the National Institute of Standards and Technology nor does it imply that any of the materials, instruments or equipment identified are necessarily the best available for the purpose.

\section{References}

[1] J.M. Butler, R. Schoske, P.M. Vallone, J.W. Redman, M.C. Kline, Allele frequencies for 15 autosomal STR loci on U.S. Caucasian, African American, and Hispanic populations, J. Forensic Sci. 48 (2003) 908-911.

[2] S.A. Miller, D.D. Dykes, H.F. Polesky, A simple salting out procedure for extracting DNA from human nucleated cells, Nucl. Acids Res. 16 (1988) 1215.

[3] V.L. Singer, L.J. Jones, S.T. Yue, R.P. Haugland, Characterization of PicoGreen reagent and development of a fluorescence-based solution assay for double-stranded DNA quantitation, Anal. Biochem. 249 (1997) 228-238.

[4] P.A. Bell, S. Chaturvedi, C.A. Gelfand, C.Y. Huang, M. Kochersperger, R. Kopla, F. Modica, M. Pohl, S. Varde, R. Zhao, X. Zhao, M.T. Boyce-Jacino, A. Yassen, SNPstream ${ }^{\circledR}$ UHT: Ultra-high throughput SNP genotyping for pharmacogenomics and drug discovery, Biotech. Suppl. (2002) 70-77.

[5] K. Liu, S. Muse, PowerMarker: new genetic data analysis software. Version 3.07. Free program distributed by the author over the internet from http://www.powermarker.net/.

[6] B. Budowle, T.R. Moretti, A.L. Baumstark, D.A. Defenbaugh, K.M. Keys, Population data on the thirteen CODIS core short tandem repeat loci in African Americans, U.S. Caucasians, Hispanics, Bahamians, Jamaicans, and Trinidadians, J. Forensic Sci. 44 (1999) 1277-1286.

[7] P. Gill, L. Foreman, J.S. Buckleton, C.M. Triggs, H. Allen, A comparison of adjustment methods to test the robustness of an STR DNA database comprised of 24 European populations, Forensic Sci. Int. 131 (2003) 184-196.

[8] R. Chakraborty, Sample size requirements for addressing the population genetic issues of forensic use of DNA typing, Hum. Biol. 64 (1992) 141-159.

[9] Council Report (NRC II), The Evaluation of Forensic DNA Evidence, Washington, DC, 1996. 
[10] J.J. Sanchez, C. Borsting, C. Hallenberg, A. Buchard, A. Hernandez, N. Morling, Multiplex PCR and minisequencing of SNPs-a model with 35 Y chromosome SNPs, Forensic Sci. Int. 137 (2003) 74-84.

[11] P. Gill, D.J. Werrett, B. Budowle, R. Guerrieri, An assessment of whether SNPs will replace STRs in national DNA data- bases-joint considerations of the DNA working group of the European Network of Forensic Science Institutes (ENFSI) and the Scientific Working Group on DNA Analysis Methods (SWGDAM), Sci. Justice 44 (2004) 51-53.

[12] P. Lincoln, A. Carracedo, Publication of population data of human polymorphisms, Forensic Sci. Int. 110 (2000) 3-5. 